\title{
Approximating a topological section by a piecewise-linear section
}

\author{
COLIN ROURKE
}

\begin{abstract}
In answer to a question from János Kollár, we prove that a surjective piecewise-linear map with a topological section admits a nearby homotopic piecewise-linear section.
\end{abstract}

57Q55; 57Q05, 57Q37

Dedicated to the memory of Christopher Zeeman, father of PL topology

Theorem Suppose $f: X \rightarrow Y$ is a piecewise-linear (PL) map between compact polyhedra and that $s: Y \rightarrow X$ is a topological section (ie a map such that $f \circ s=\operatorname{id}_{Y}$ ). Then for each $\varepsilon>0$ there is a PL section $q: Y \rightarrow X$ and an $\varepsilon$-homotopy through sections from $s$ to $q$.

Further if $s$ is already PL on an open neighbourhood $N$ of a closed subset $Z$ of $Y$, then we can assume that, restricted to some smaller open neighbourhood, it is not moved during the homotopy.

The essence of the proof is to use conical extension (using the fact that a PL map is locally conical) and the Alexander trick for the homotopy. The construction is by induction over the skeleta of a triangulation of $Y$ and the method makes both the relative version and the smallness of the homotopy clear. The result can be extended to locally compact polyhedra by a straightforward union argument.

Proof The proof is by double induction with the primary induction being on the dimension of $Y$. Assume $Y$ has dimension $n$ and that the theorem holds when $Y$ has dimension less than $n$.

By standard PL theory, Rourke and Sanderson [4], we can triangulate $f$, ie find simplicial complexes $|K|=X$ and $|L|=Y$ so that $f: K \rightarrow L$ is simplicial. We use a secondary induction on $i$ to construct the required section and homotopy on a neighbourhood of the $i$-skeleton of $Y$. Inductively, we assume that $s$ is homotopic through sections to $s_{i-1}$, which is PL in a neighbourhood of the $(i-1)$-skeleton, by a homotopy fixed on a neighbourhood of $Z$, and we construct a homotopy through sections of $s_{i-1}$ to $s_{i}$ which is PL in a neighbourhood of the $i$-skeleton. Further 
this homotopy fixes a (possible smaller) neighbourhood of the $(i-1)$-skeleton, the complement of a neighbourhood of the $i$-skeleton and a neighbourhood of $Z$. We only need to consider one particular $i$-simplex $\Delta$ during the construction because, once a neighbourhood of the $(i-1)$-skeleton is excised, the $i$-simplices are all disjoint. For simplicity of notation write $s$ for $s_{i-1}$.

Let $D$ be a concentric smaller simplex such that the section is already PL on a neighbourhood of the closure of $\Delta-D$. We need to extend the PL section to a neighbourhood of $D$. Later we shall be more specific about the choice of $D$. We now digress to describe the structure of $f$ over $D$ in detail. Not all this structure is used in the proof, but it seemed worth giving all the detail.

Digression on the structure of $\boldsymbol{f}$ over $\boldsymbol{D}$ Over $D$ the map $f$ is the projection of a PL product $D \times F \rightarrow D$, where $F$ is a compact polyhedron. This is a basic property of simplicial maps, which can be seen as follows. $f^{-1}(\Delta)$ is a collection of simplexes, each of which maps to $\Delta$ as a "squeezed" product, which is a true product when restricted to $f^{-1}(D)$. More precisely, let $\Lambda$ be a simplex of dimension $\ell$ in $f^{-1}(\Delta)$, then $f^{-1}(D) \cap \Lambda$ is PL isomorphic to $D \times A_{\Lambda}$, where $A_{\Lambda}$ is a cell of dimension $\ell-i$. Choose a point $x \in D$ then the cells $\left\{\{x\} \times A_{\Lambda} \mid \Lambda \in f^{-1}(\Delta)\right\}$ fit together like the corresponding simplexes of $f^{-1}(\Delta)$ to form a polyhedron $F$ and $f^{-1}(D) \cong D \times F$ over $D$; see Figure 1 .
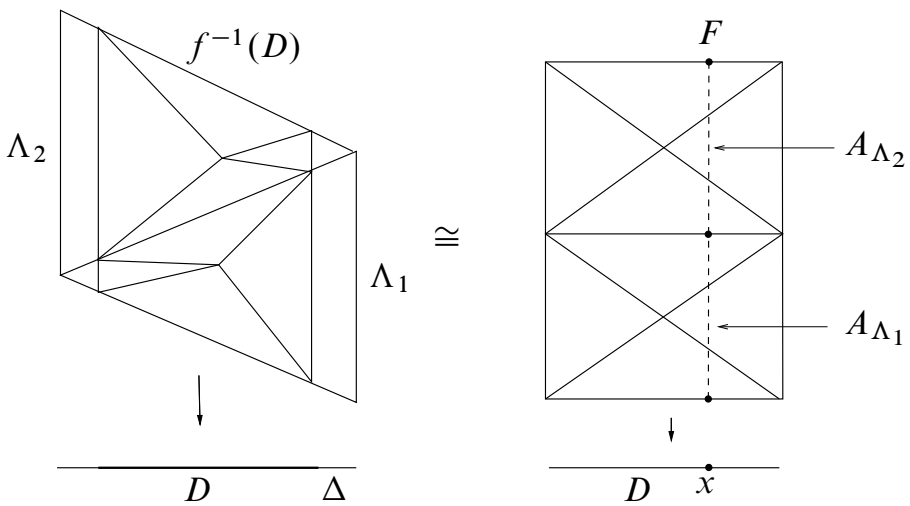

Figure 1: The product structure over $D$

There is a similar product structure for an $\varepsilon$-neighbourhood of $\Delta$ in $Y$ restricted to $D$. Let $\Delta<\Lambda \in L$, ie $\Lambda$ is a simplex (of dimension $\ell$ say) of which $\Delta$ is a face, then the $\varepsilon$-neighbourhood of $\Delta$ in $\Lambda$ is a PL product $\Delta \times B_{\Lambda}$ where $B_{\Lambda}$ is a cell of dimension $\ell-i$. The cells $\left\{\{x\} \times B_{\Lambda} \mid \Delta<\Lambda\right\}$ fit together to form the dual cone $C$ at $x$ and the products fit together over $D$ to give a PL embedding of $D \times C \rightarrow Y$ with image $N$ say which is a neighbourhood of $\operatorname{int}(D)$ in $Y$; see Figure 2. 


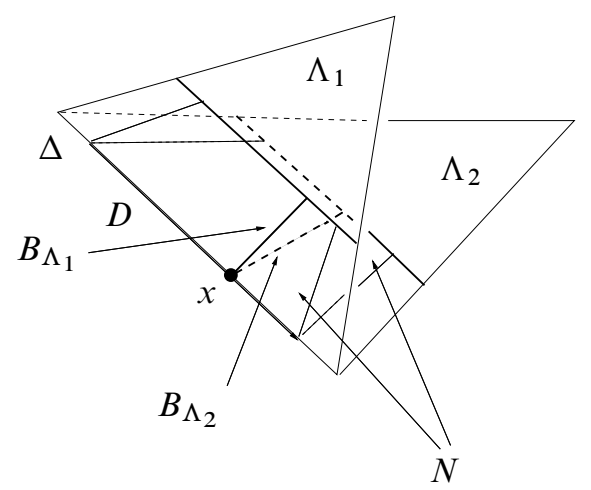

Figure 2: The dual cone $C=\bigcup_{i} B_{\Lambda_{i}}$ and product neighbourhood $N \cong D \times C$

Now let $M=f^{-1}(C)$ then by a similar analysis to the above proof that $f^{-1}(D) \cong$ $D \times F$ we can see that $f^{-1}(D \times C) \cong D \times M$ over $D$. There is a pull-back diagram

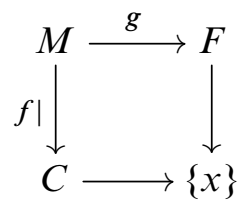

which defines the projection $g: M \rightarrow F$. We have used the notation $M$ because $g$ is in fact the projection of a (simplicial) mapping cylinder, though we shall not need to use this fact. The product structures found above extend to the whole diagram. In other words we have a diagram like this for each point $x$ of $D$ with the collection isomorphic to the product of one diagram with $D$. (These facts are a special case of the general structure of a PL map described in Marshall Cohen's PhD thesis [2].) This ends the digression.

Proof of theorem continued From now on we shall identify these product structures with their images and, for example, speak of $D \times C$ as a subset of $Y$. We can now be specific about the choice of $D$. We need that $\partial D \times C$ is contained in the neighbourhood of the $(i-1)$-skeleton where the PL section is already defined and we choose $D$ with $\partial D$ close enough to $\partial \Delta$ and $C$ sufficiently small for this to happen. Later we shall need to make $C$ smaller still.

From the description of the structure given above, $f$ is locally conical. Indeed this is one of the equivalent definitions of a PL map. In other words for each $y \in F$ there is a conical neighbourhood $T$ with vertex $y$ in $M$ and a conical neighbourhood of $f(y)$ in $C$ (ie a smaller cone which we also call $C$ ) such that $f \mid T$ is a conical map. Furthermore if we replace $C$ by an even smaller cone $C^{\prime}$ and $T$ by its preimage, $f \mid$ 
remains conical and $T \cap F$ is unchanged. Denote this preimage $T \mid C^{\prime}$. Choose an open cover $\mathcal{O}$ of $F$ by conical neighbourhoods of this type (ie $T \cap F$ for conical $T$ mapping to $C$ sufficiently small) and then use continuity to find a $\delta>0$ and a possibly smaller subcone $C^{\prime}$ of $C$ such that:

(*) For each set $Q \subset D$ of diameter $<\delta, s\left(Q \times C^{\prime}\right) \subset Q \times T \mid C^{\prime}$ for some $T \cap F \in \mathcal{O}$.

Now triangulate $D$ by simplices of diameter smaller than $\delta$. Construct the PL section $s_{i}$ (and homotopy from $s$ ) on $D \times C^{\prime}$ as follows. Using the primary induction hypothesis we can assume that $s_{i}$ and the homotopy are already defined on the $(i-1)$-skeleton of this triangulation $\times C^{\prime}$ and on $D \times \beta C^{\prime}$, where $\beta$ denotes the base of the cone and that these extend those already found (or given) near the $(i-1)$-skeleton of $Y$ and near $Z$. Further by choosing the homotopy sufficiently small in the call to induction we can assume that property $(*)$ is not disturbed (where $s\left(Q \times C^{\prime}\right)$ is replaced by $s_{i}(R)$ with $R \subset Q \times C^{\prime}$ the subset where $s_{i}$ is defined at this point).

We extend the PL section, and the homotopy from $s$, to $\sigma \times C^{\prime}$ for each $i$-simplex $\sigma$. We note that $\sigma \times C^{\prime}$ is a cone from the barycentre $\hat{\sigma}$ with base

$$
B=\partial \sigma \times C^{\prime} \cup \sigma \times \beta C^{\prime} .
$$

Now $s$ maps $\sigma \times C^{\prime}$ to a cone $U$ of the form $\sigma \times T \mid C^{\prime}$ with $s(B) \subset \beta U$. Further we can take the cone point $u$ of $U$ to lie over $\hat{\sigma}$.

Use the Alexander trick (see note below) to homotope $s \mid \sigma \times C^{\prime}$ to the cone on $s \mid B$, and then extend the homotopy of $s \mid B$ conically to $s \mid \sigma \times C^{\prime}$. The final map is the cone on a PL map and hence PL. (These two homotopies can be combined, if desired, with the Alexander trick homotopy and the conical extension carried out simultaneously.)

At this point, $s_{i}$ and the homotopy from $s$ are defined on a neighbourhood of $Z \cup(i-1)-$ skeleton, together with $D \times C^{\prime}$, which all forms a neighbourhood of the $i$-simplex $\Delta$. A similar definition works for all the $i$-simplexes and hence $s_{i}$ is defined on a neighbourhood of the $i$-skeleton. We need to extend (not necessarily PL) to the rest of $Y$. But the frontier of this neighbourhood where the homotopy has been altered is a subset of $D \times \beta C^{\prime}$ which is collared in $Y$ and therefore the homotopy extends to the outside by a simple collar extension argument. This completes the secondary induction step.

It remains to comment that the relative version can be proved by choosing the triangulation of $D$ to contain a simplicial neighbourhood of $Z \cap D$ inside $N$ and leaving the section unmoved in this neighbourhood, and that the smallness is guaranteed by choosing the sets of the cover $\mathcal{O}$ sufficiently small. 
Note on the Alexander trick The "Alexander trick" [1] is an homotopy defined for any map $g: A \rightarrow B$ between cones which takes $\beta A$ to $\beta B$. Let $A_{t}$ for $0 \leq t \leq 1$ be the subcone extending $t$ of the way from the cone point. At the $(1-t)^{\text {th }}$ stage, map $A_{t}$ to $B_{t}$ by the starting map shrunk by factor $t$ and map the rest by mapping cone lines isometrically. When $t=0$ we have the original map $g$ and when $t=1$ we have the cone on $g \mid \beta A$. And notice that we did not need to assume that the original map took the cone point of $A$ to the cone point of $B$. If $g$ is a section of a conical map $f: B \rightarrow A$ then it can be checked that the Alexander trick is an homotopy through sections of $f$.

Remark János Kollár asked me by email on 30 August 2010 whether the result proved here was true. It seemed obvious and easy, but I could not find anything that was proved in the "golden age" of PL topology that implied it, nor could I find a PL topologist who knew a proof. It was surprisingly hard to find a proof. I thought at first that the proof of the simplicial approximation theorem (see eg Hatcher [3, 2C1]) could be adapted, but this turned out to be fruitless. What is needed is a fibrewise version of the theorem and the standard proof will not work in a fibred setting. You can regard the result proved here as being the fibrewise version of the theorem.

\section{References}

[1] J W Alexander, On the deformation of an n-cell, Proc. Nat. Acad. Sci. USA 9 (1923) 406-407

[2] M M Cohen, Simplicial structures and transverse cellularity, Ann. of Math. 85 (1967) 218-245 MR0210143

[3] A Hatcher, Algebraic topology, Cambridge Univ. Press (2002)

[4] C P Rourke, B J Sanderson, Introduction to piecewise-linear topology, Ergeb. Math. Grenzgeb. 69, Springer, New York (1972)

Mathematics Institute, University of Warwick

Coventry CV4 7AL, UK

cpr@msp.warwick.ac.uk

http://msp.warwick.ac.uk/ cpr

Received: 31 January 2014 Revised: 10 September 2015 
